\title{
Estudo sobre o piritoedro
}

\author{
Roberto Ribeiro Paterlini (1)
}

\begin{abstract}
Resumo
O piritoedro é um dodecaedro exclusivamente com faces pentagonais, congruentes duas a duas, não regulares, com uma forma específica que descrevemos abaixo. Com a publicação desse texto esperamos prover o professor de Matemática do Ensino Médio com informações e ideias para construir uma sequência didática que permita aos seus estudantes tomarem conhecimento desse interessante poliedro. Ao mesmo tempo o professor poderá praticar a interdisciplinaridade com a Mineralogia, já que o piritoedro é modelo de um tipo de pirita, um mineral relativamente bem conhecido. Os estudantes poderão fazer, a partir dessas atividades, muitos outros contatos matemáticos com essa antiga e fantástica ciência, uma fonte clássica de inspiração para a Geometria.
\end{abstract}

Palavras-chave: piritoedro; poliedros; Geometria Espacial; Mineralogia.

\begin{abstract}
The pyritohedron is a dodecahedron exclusively with pentagonal faces, congruent two by two, nonregular, with a specific shape that we describe below. With the publication of this text we hope to provide the high school mathematics teacher with information and ideas to build a didactic sequence that allows its students to become aware of this interesting polyhedron. At the same time, the teacher will be able to practice interdisciplinarity with Mineralogy, since the pyrite is a model of a type of pyrite, a relatively well-known mineral. Students will be able to make, from these activities, many other mathematical contacts with this ancient and fantastic science, a classic source of inspiration for Geometry.
\end{abstract}

Keywords: pyritohedron; polyhedra; Spatial Geometry; Mineralogy.

\section{Introdução}

Conforme já comentamos, o nome "piritoedro" advém do mineral pirita, o qual, em uma de suas formas, tem como modelo o dodecaedro com faces pentagonais (existe também pirita nas formas cúbica ou octaédrica). Para obter mais detalhes confira, por exemplo, a referência [7].

Vemos, na Figura 1, uma foto de uma pirita dodecaédrica, e, na Figura 2, um modelo do piritoedro. Nesse modelo as arestas de uma mesma cor são congruentes entre si, mas de medida diferente das arestas da outra cor. 


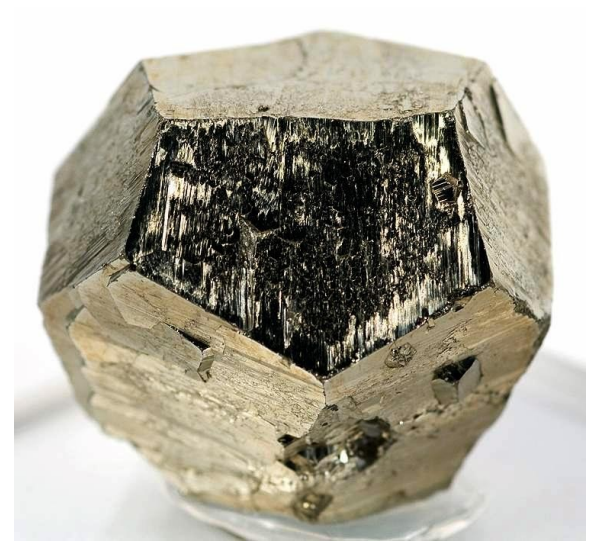

Figura 1: Foto do mineral pirita em formato dodecaédrico. Autoria: Lavinsky, R.M. [3]

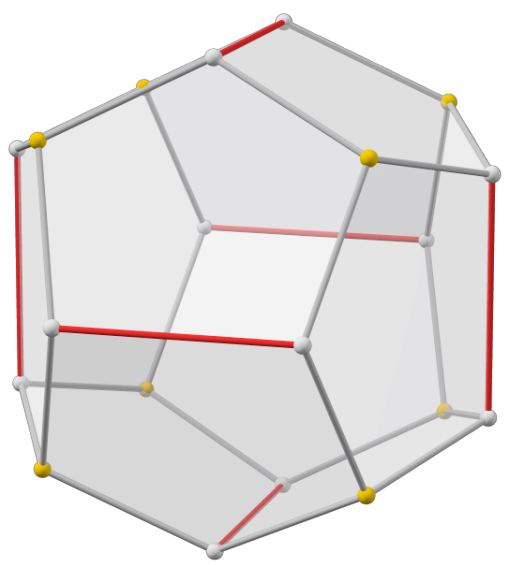

Figura 2: Piritoedro. Autoria: Watchduck [5].

Dodecaedros são poliedros com 12 faces. O mais conhecido é o dodecaedro regular, um dos sólidos de Platão, constituído por faces pentagonais regulares apenas. Em geral, um dodecaedro pode ter faces de vários tipos. Para ver diversos modelos consulte, por exemplo, [1].

No dodecaedro regular a quantidade de arestas é 30 e a de vértices, 20. Todos os vértices são do tipo três, isto é, a cada um deles concorrem exatamente três arestas (ou três faces).

O piritoedro tem essa mesma configuração combinatória, mas as faces são pentágonos não regulares de um certo formato, que passamos a descrever.

\section{Construção de modelos}

Modelos de piritoedros podem ser feitos de papel-cartão. Para isso precisamos de medidas que permitam o recorte das faces de forma correta.

Primeiro escolhemos um valor d >0, que será fixo. O valor $2 \mathrm{~d}$ é a medida de uma diagonal da face, e, de certo modo, determina o "tamanho" do modelo. Confira a Figura 3. Em seguida escolhemos um valor h, variável, com $0<\mathrm{h}<\mathrm{d}$. Esse valor é a altura comum dos seis "telhados" que compõem o poliedro. Um dos lados do pentágono é paralelo à referida diagonal, e tem medida, digamos, b. Esse lado será denominado base do pentágono. O valor b é dependente de h (e de d) segundo a fórmula:

$$
\mathrm{b}=\frac{2}{\mathrm{~d}}\left(\mathrm{~d}^{2}-\mathrm{h}^{2}\right)
$$

Os outros lados do pentágono têm todos medida $\ell$, guardando relação com h (e com d) segundo a fórmula:

$$
\ell=\frac{1}{d} \sqrt{d^{4}+h^{4}+d^{2} h^{2}}
$$


O pentágono é simétrico em relação à mediatriz da base. Para fazer o desenho do pentágono é útil sabermos sua altura a em relação à base. Ela é dada pela fórmula:

$$
a=\frac{d+h}{d} \sqrt{d^{2}+h^{2}}
$$

Se $h=\frac{d}{2}(-1+\sqrt{5})$, então o poliedro é o dodecaedro regular.

Essas relações serão explicadas na próxima seção. Para facilidade dos estudantes, apresentamos abaixo um desenho de um pentágono que pode ser usado como face, assim como uma planificação do piritoedro construído com faces com essas medidas (em escala reduzida). No desenho dessa face escolhemos $\mathrm{d}=3 \mathrm{~cm}$ e $\mathrm{h}=2 \mathrm{~cm}$. A distância entre a diagonal e a base é $\approx 3,61 \mathrm{~cm}$.

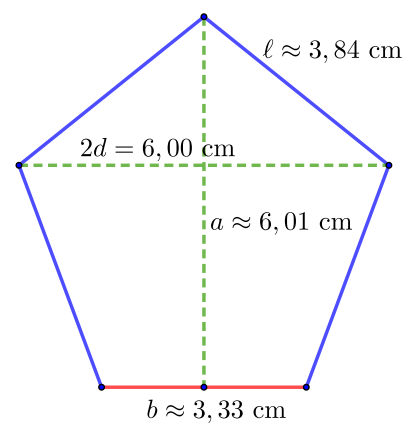

Figura 3: Face pentagonal de um piritoedro.

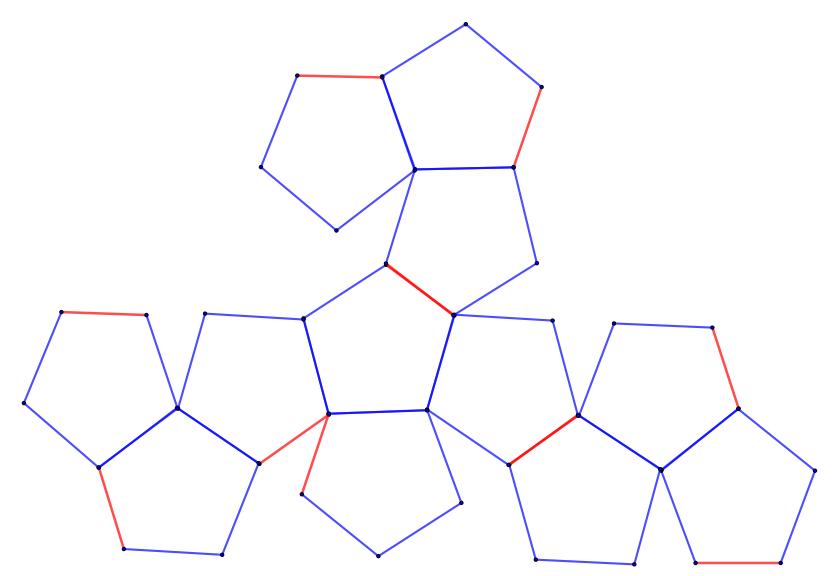

Figura 4: Uma planificação de um piritoedro.

\section{Justificativas para as medidas do piritoedro}

Começamos com a observação de que o dodecaedro regular tem um cubo inscrito, cujas arestas são certas diagonais de suas faces. O dodecaedro é constituído pelo ajuntamento de seis "telhados" congruentes às faces desse cubo.

A mesma ideia será usada para a construção dos piritoedros. Começamos com um cubo ABCD EFGH, que será fixo no decorrer dessa construção, com aresta 2d. O cubo está desenhado na Figura 5. A mesma figura mostra um telhado ABCD - IJ. Trata-se de um poliedro com base ABCD (portanto coincidente com uma das faces do cubo). Outras duas faces são ADI e BCJ, triângulos isósceles congruentes de bases $\mathrm{AD}$ e $\mathrm{BC}$, respectivamente. As duas faces restantes são os trapézios congruentes ABJI e CDIJ. A Figura 5 mostra ainda outro telhado BCGF - KL. No total temos seis telhados, congruentes dois a dois, um para cada face do cubo. O topo de um telhado é a aresta de encontro das faces trapezoidais, e sua altura h é a distância do topo à sua base (observando que o topo é paralelo a essa base). 
Para que a construção dê certo, as faces BCJ e BCLK precisam ser coplanares, de modo que possam constituir uma face pentagonal plana do futuro piritoedro. Para deteminar isso, pensamos que a melhor forma seja colocar a figura em um sistema de coordenadas cartesianas, e encontrar as coordenadas de todos os seus vértices.

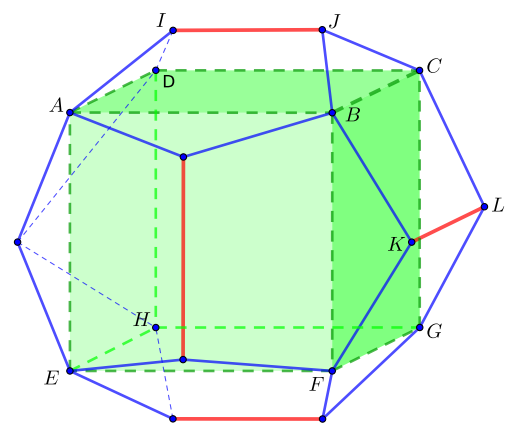

Figura 5: Cubo e telhados do piritoedro.

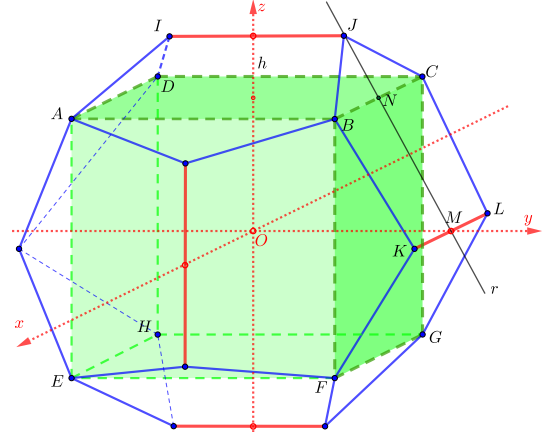

Figura 6: Piritoedro em um sistema de coordenadas.

A Figura 6 mostra um sistema de coordenadas Oxyz com origem no centro do cubo, e com os eixos passando pelos centros das suas faces. Observamos ainda que os eixos passam pelos pontos médios dos topos dos telhados.

As coordenadas dos vértices do cubo (que são também coordenadas de oito dos vértices do futuro piritoedro) são:

$$
\begin{array}{cccc}
\mathrm{A}=(\mathrm{d},-\mathrm{d}, \mathrm{d}) & \mathrm{B}=(\mathrm{d}, \mathrm{d}, \mathrm{d}) & \mathrm{C}=(-\mathrm{d}, \mathrm{d}, \mathrm{d}) & \mathrm{D}=(-\mathrm{d},-\mathrm{d}, \mathrm{d}) \\
\mathrm{E}=(\mathrm{d},-\mathrm{d},-\mathrm{d}) & \mathrm{F}=(\mathrm{d}, \mathrm{d},-\mathrm{d}) & \mathrm{G}=(-\mathrm{d}, \mathrm{d},-\mathrm{d}) & \mathrm{H}=(-\mathrm{d},-\mathrm{d},-\mathrm{d})
\end{array}
$$

Como KL $\| \mathrm{BC}$, a reta $\mathrm{r}$ que passa pelo ponto médio $\mathrm{M}$ de $\mathrm{KL}$ e pelo ponto médio de $\mathrm{BC}$ deve encontrar $\mathrm{J}$ (para que JBKLC seja um pentágono plano, conforme já observamos). Temos $\mathrm{M}=$ $(0, d+h, 0)$ e $\mathrm{N}=(0, d, d)$. Portanto, um vetor direcional de $\mathrm{r}$ é $\overrightarrow{\mathrm{u}}=\mathrm{N}-\mathrm{M}=(0,-\mathrm{h}, \mathrm{d})$. Uma equação para os pontos $(\mathrm{x}, \mathrm{y}, \mathrm{z})$ de $\mathrm{r}$ é:

$$
(\mathrm{x}, \mathrm{y}, \mathrm{z})=\mathrm{M}+\mathrm{tu}=(0, \mathrm{~d}+\mathrm{h}, 0)+\mathrm{t}(0,-\mathrm{h}, \mathrm{d})=(0, \mathrm{~d}+\mathrm{h}-\mathrm{th}, \mathrm{td}), \quad \mathrm{t} \in \mathbb{R}
$$

A reta $\mathrm{r}$ encontra JI em J. Esse segmento está no plano $\mathrm{x}=0$ e seus pontos têm alturas $\mathrm{d}+\mathrm{h}$. Portanto em $J$ devemos ter $t d=d+h$, ou $t=(d+h) / d$. Assim,

$$
J=\left(0, d+h-\frac{d+h}{d} h, \frac{d+h}{d} d\right)=\left(0, \frac{1}{d}\left(d^{2}-h^{2}\right), d+h\right)
$$

Usando simetria e cálculos análogos, temos

$$
I=\left(0,-\frac{1}{d}\left(d^{2}-h^{2}\right), d+h\right) \quad K=\left(\frac{1}{d}\left(d^{2}-h^{2}\right), d+h, 0\right) \quad L=\left(-\frac{1}{d}\left(d^{2}-h^{2}\right), d+h, 0\right)
$$


Repetindo cálculos e usando simetria vemos que as coordenadas dos 12 vértices do piritoedro que são extremos de topos de telhados são

$$
\left(0, \pm \frac{1}{d}\left(d^{2}-h^{2}\right), \pm(d+h)\right) \quad\left( \pm \frac{1}{d}\left(d^{2}-h^{2}\right), \pm(d+h), 0\right) \quad\left( \pm(d+h), 0, \pm \frac{1}{d}\left(d^{2}-h^{2}\right)\right)
$$

Os quatro primeiros vértices correspondem aos extremos do topo IJ e seu simétrico no plano Oyz. Os outros quatro correspondem aos extremos do topo KL e seu simétrico no plano Oxy. Por fim os quatro últimos são os extremos dos dois topos no plano Oxz.

Notemos que o valor $\frac{1}{\mathrm{~d}}\left(\mathrm{~d}^{2}-\mathrm{h}^{2}\right)$, distância de $\mathrm{J}$ ao plano Oxz, é a metade do topo, portanto é a metade da base da face. Assim,

$$
\mathrm{b}=\frac{2}{\mathrm{~d}}\left(\mathrm{~d}^{2}-\mathrm{h}^{2}\right)
$$

confirmando a fórmula (1).

Vejamos agora que $\mathrm{JB}=\mathrm{BK}$. Temos

$$
\begin{aligned}
J B=|B-J|= & \left|(d, d, d)-\left(0, \frac{1}{d}\left(d^{2}-h^{2}\right), d+h\right)\right|=\left|\left(d, \frac{h^{2}}{d},-h\right)\right|= \\
& =\sqrt{d^{2}+\frac{h^{4}}{d^{2}}+h^{2}}=\frac{1}{d} \sqrt{d^{4}+h^{4}+d^{2} h^{2}}
\end{aligned}
$$

e

$$
B K=|K-B|=\left|\left(\frac{1}{d}\left(d^{2}-h^{2}\right), d+h, 0\right)-(d, d, d)\right|=\left|\left(-\frac{h^{2}}{d}, h,-d\right)\right|=J B
$$

Temos assim, por simetria, que $\mathrm{JB}=\mathrm{BK}=\mathrm{CL}=\mathrm{JC}$, e lembramos que esse é o valor $\ell$ comum aos lados do pentágono diferentes da base. Portanto,

$$
\ell=\frac{1}{d} \sqrt{d^{4}+h^{4}+d^{2} h^{2}}
$$

confirmando a fórmula (2).

Para confirmar a altura do pentágono dada na fórmula (3), calculamos

$$
a=J M=\frac{d+h}{d} \sqrt{d^{2}+h^{2}}
$$

Vejamos agora que se $\mathrm{h}=\frac{\mathrm{d}}{2}(-1+\sqrt{5})$, então o piritoedro é, na verdade, o dodecaedro regular. Apresentamos os cálculos resumidamente.

Primeiro verificamos que o pentágono é equilátero. Temos

$$
\mathrm{b}=\frac{2}{\mathrm{~d}}\left(\mathrm{~d}^{2}-\mathrm{h}^{2}\right)=\mathrm{d}(\sqrt{5}-1)
$$


e

$$
\ell=\frac{1}{d} \sqrt{d^{4}+h^{4}+d^{2} h^{2}}=d \sqrt{6-2 \sqrt{5}}=d \sqrt{(\sqrt{5}-1)^{2}}=d(\sqrt{5}-1)
$$

Portanto $\mathrm{b}=\ell$, e o pentágono é equilátero.

Devemos agora provar que o pentágono é equiangular. Para evitar calcular ângulos, vamos verificar que a razão entre a altura (em relação à base) e o lado coincide com o valor preconizado para o pentágono regular, que é $\frac{1}{2} \sqrt{5+2 \sqrt{5}}$. Como essa altura está na mediatriz da base, isso é suficiente para determiná-lo.

Primeiro simplificamos a fórmula $(3)$ usando $h=\frac{d}{2}(-1+\sqrt{5})$. Temos (resumidamente)

$$
\begin{gathered}
a=\frac{d+h}{d} \sqrt{h^{2}+d^{2}}=(d+h) \sqrt{\frac{h^{2}}{d^{2}}+1}=\left[d+\frac{d}{2}(-1+\sqrt{5})\right] \sqrt{\frac{1}{4}(-1+\sqrt{5})^{2}+1} \\
\Rightarrow a=\frac{\sqrt{2} d}{2} \sqrt{5+\sqrt{5}}
\end{gathered}
$$

Portanto,

$$
\begin{gathered}
\frac{\mathrm{a}}{\ell}=\frac{\sqrt{2} \sqrt{5+\sqrt{5}}}{2(\sqrt{5}-1)}=\frac{1}{8} \sqrt{2} \sqrt{(5+\sqrt{5})(1+\sqrt{5})^{2}}= \\
=\frac{1}{8} \sqrt{2} \sqrt{40+16 \sqrt{5}}=\frac{1}{2} \sqrt{5+2 \sqrt{5}}
\end{gathered}
$$

o que termina a verificação.

Escolhendo os valores $\mathrm{d}>0$ e $0<\mathrm{h}<\mathrm{d}$ e calculando $\ell$, b e a, a face pentagonal pode ser desenhada usando-se régua e compasso ou um aplicativo para Geometria.

Observação 1. Alguns textos, como [6], trazem as coordenadas do piritoedro calculadas para $\mathrm{d}=1$. Nesse caso os vértices são $( \pm 1, \pm 1, \pm 1),\left(0, \pm\left(1-\mathrm{h}^{2}\right), \pm(1+\mathrm{h})\right),\left( \pm\left(1-\mathrm{h}^{2}\right), \pm(1+\mathrm{h}), 0\right) \mathrm{e}$ $\left( \pm(1+\mathrm{h}), 0, \pm\left(1-\mathrm{h}^{2}\right)\right)$, com $0<\mathrm{h}<1$. A base do pentágono é $\mathrm{b}=2\left(1-\mathrm{h}^{2}\right)$ e o lado, $\ell=$ $\sqrt{1+h^{2}+h^{4}}$. A altura do pentágono em relação à base é $a=(1+h) \sqrt{1+h^{2}}$.

Em [2] o leitor pode ver as diversas formas que o piritoedro assume com a variação de $h$. Se $h<0$, o poliedro é não convexo.

Observação 2. Na Figura 7 vemos foto de um modelo de piritoedro construído pelo autor segundo as medidas da Figura 3. O modelo está apoiado em um topo, em posição similar à da Figura 5.

Observação 3. Outra família interessante de dodecaedros não regulares e com faces pentagonais são os tetartoides. Instruções para construir modelos podem ser encontradas em [4], págs. 475 e 476. Consulte também [6]. 


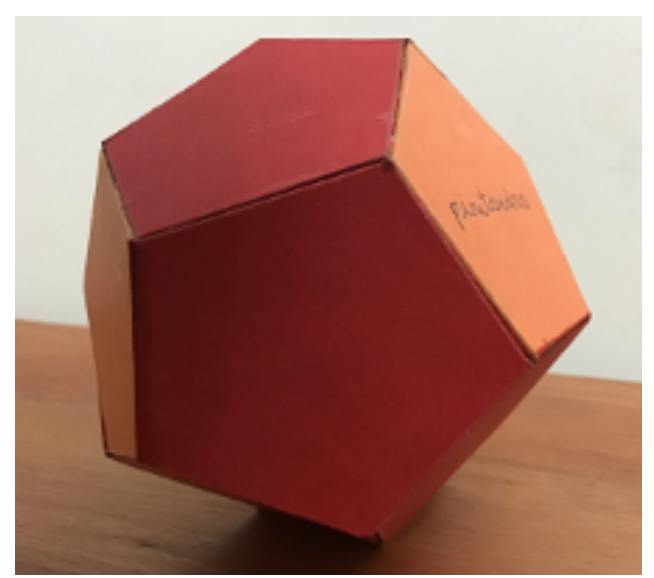

Figura 7: Piritoedro. Foto do autor.

\section{Referências}

[1] Altes, G. K. Modelos de papel de poliedros. https://www.polyhedra.net/es Consultado em 21 de março de 2020. Entre no item "Ordinati per numero di facce" ou/e em "indice alfabetico", e, em seguida, acesse "dodecaedro".

[2] Campuzano, J. C. P. Pyritohedron. In Repositório Geogebra. https://www.geogebra.org/m/ qbsfrf8e Consultado em 21 de março de 2020.

[3] Lavinsky, R. M. Pyrita. Rob Lavinsky, iRocks.com - CC-BY-SA-3.0. Disponível em https://commons.wikimedia.org/wiki/File:Pyrite-193871.jpg sob licença Creative Commons Attribution-Share Alike 3.0 Unported. Consultado em 20 de março de 2020.

[4] Paterlini, R. R. "A família dos poliedros fulerenos". Professor de Matemática Online, Vol. 8, n⿳0 5, págs. 470-489. http://pmo.sbm.org.br/wp-content/uploads/sites/16/dlm_uploads/2020/ 09/art34_vol8_PMO_SBM_2020.pdf Consultado em 14 de jun de 2021.

[5] Watchduck. Piritoedro. Disponível em https://commons.wikimedia.org/wiki/File:Polyhedron pyritohedron_transparent_max.png sob licença Creative Commons Attribution-Share Alike 3.0 Unported. Consultado em 20 de março de 2020.

[6] Wikipedia. Dodecahedron. Disponível em https://en.wikipedia.org/wiki/Dodecahedron Consultado em 20 de março de 2020.

[7] Wikipedia. Pirita. Disponível em https://pt.wikipedia.org/wiki/Pirita Consultado em 21 de março de 2020.

Roberto Ribeiro Paterlini Universidade Federal de São Carlos <paterlini@ufscar.br>

Recebido: $16 / 06 / 2021$

Publicado: 30/11/2021 